This item was submitted to Loughborough's Research Repository by the author.

Items in Figshare are protected by copyright, with all rights reserved, unless otherwise indicated.

\title{
Sensitivity improvement of an optical current sensor with enhanced Faraday
} rotation

PLEASE CITE THE PUBLISHED VERSION

PUBLISHER

(c) IEEE

VERSION

VoR (Version of Record)

LICENCE

CC BY-NC-ND 4.0

\section{REPOSITORY RECORD}

Li, Gongde, Michael G. Kong, Gordon R. Jones, and Joe W. Spencer. 2019. "Sensitivity Improvement of an Optical Current Sensor with Enhanced Faraday Rotation”. figshare. https://hdl.handle.net/2134/5217. 
This item was submitted to Loughborough's Institutional Repository (https://dspace.lboro.ac.uk/) by the author and is made available under the following Creative Commons Licence conditions.

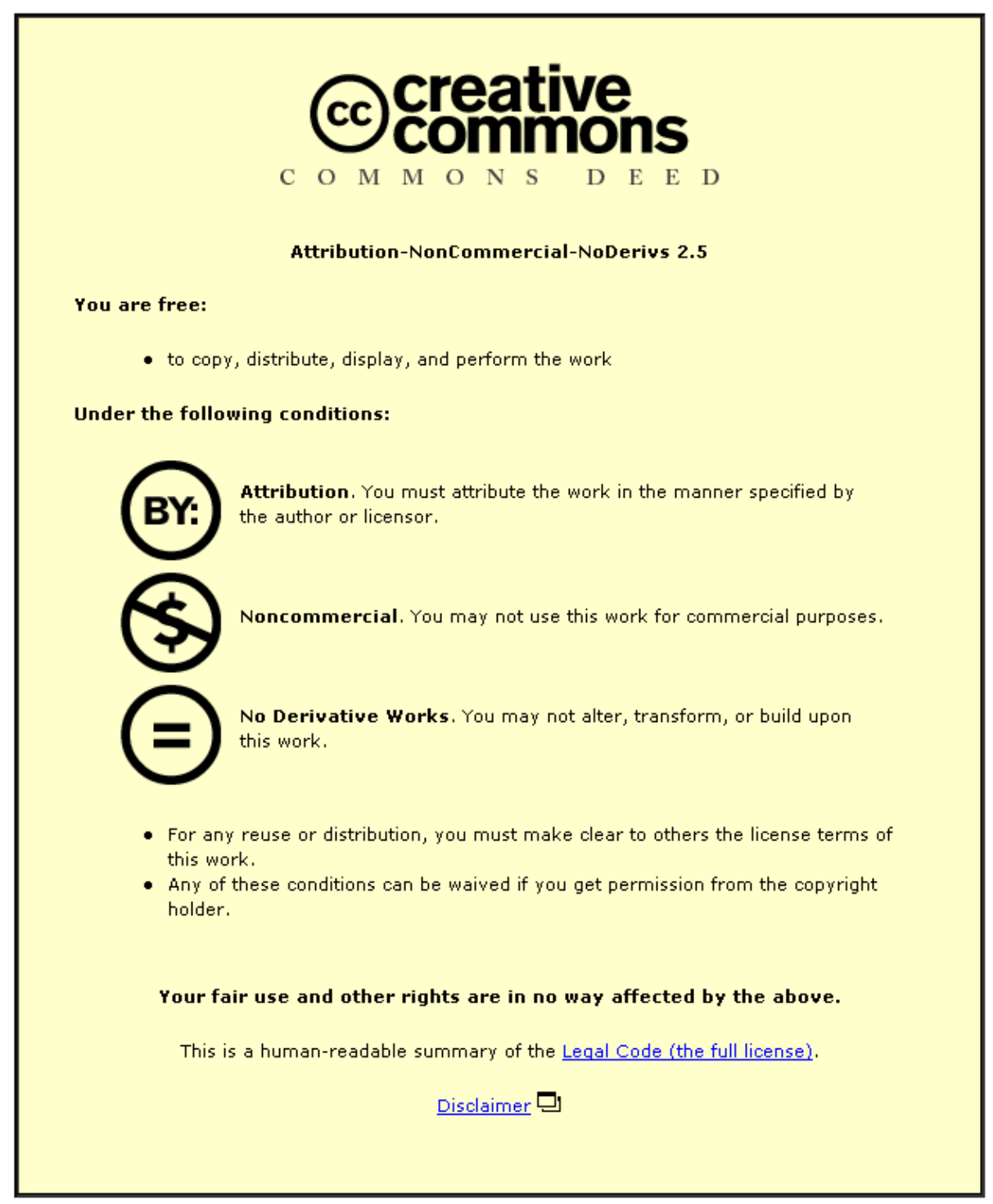

For the full text of this licence, please go to: http://creativecommons.org/licenses/by-nc-nd/2.5/ 


\title{
Sensitivity Improvement of an Optical Current Sensor with Enhanced Faraday Rotation
}

\author{
Gongde Li, Student Member, IEEE, Michael G. Kong, Member, IEEE, Gordon R. Jones, \\ and Joe W. Spencer
}

\begin{abstract}
A sensitivity improvement technique is proposed for a class of bulk-glass optical current sensors that employ a ferromagnetic field concentrator. The total effective optical path length is demonstrated theoretically to be an invariant regardless of the bulk glass thickness and consequently independent of the size of the concentrator gap opening. Thus, if the magnetic field is increased by reducing the gap size, the eventual Faraday rotation for a given electric current can be increased proportionally, leading to an improved device sensitivity. The dependence of the gap magnetic field on gap size is calculated with an equivalent circuit model, and this analytical treatment is compared with a dedicated finite element computer package. By taking account of various types of optical power losses present in the bulk glass, the above formulated gap dependence of magnetic field is used to aid a realistic assessment of device sensitivity and this serves as a tool to design and analyze practical bulk-glass optical current sensors. A detailed experimental study to confirm the proposed sensitivity improvement technique is also reported.
\end{abstract}

Index Terms-Current measurement, Faraday effect, magnetooptic transducers, optical fiber measurements, optical reflection, sensitivity.

\section{INTRODUCTION}

$\mathbf{T}$ HE measurement of electric current by means of optical methods is attractive especially for power system applications due to many desirable features such as the large inherent bandwidth and relative immunity to electromagnetic interference [1]-[8]. Among optical current sensors (OCS's) developed, one class is based on the principle of Faraday rotation [5]-[8]. The sensing element can be implemented from either a continuous length of optical fiber [9]-[15] or a bulk optical glass [16]-[22]. OCS's using optical fibers are of straightforward configuration but often suffer from a large amount of linear birefringence induced in the fiber when it is deployed. For OCS's using bulk optical glass, on the other hand, the problems associated with bend-induced birefringence do not occur [7]-[8], [23]. These devices also benefit from the higher Verdet constants of many suitable glasses than that of silica and this can usually lead to a greater sensitivity of the eventual device.

One possible arrangement for an OCS with a bulk glass is shown schematically in Fig. 1 where a ferromagnetic ring is used as a magnetic field concentrator [16], [19]-[22]. While the employment of the concentration ring introduces certain disadvantages such as lower isolation and saturation effects in

Manuscript received February 24, 1997; revised September 8, 1997.

The authors are with the Department of Electrical Engineering and Electronics, University of Liverpool, Liverpool, L69 3GJ UK.

Publisher Item Identifier S 0733-8724(97)08881-6. the ring, this type of OCS's has been widely used often in a form of commercial products. A gap is introduced in the concentration ring for the insertion of a bulk optical glass. Thus when an optical beam is fed into the glass, it is subject to the strong magnetic field in the concentrator and as a result its polarization undergoes an appreciable Faraday rotation. It is well known that the extent of Faraday rotation angle, $\theta$, is given by [24]

$$
\theta=V B L_{\mathrm{opt}}
$$

where $V$ is the Verdet constant of the glass material, $B$ is the magnetic field, and $L_{\mathrm{opt}}$ is the path length of the optical beam along the direction of the magnetic field when transversing the glass. To design an OCS of good sensitivity, a significant rotation angle at a given electric current needs to be produced. This may be achieved by producing a sizeable $B L_{\text {opt }}$ product as indicated in (1).

One possible technique to enhance the $B L_{\mathrm{opt}}$ product for a given electric current is to reduce the gap size of the concentrator so that the magnetic field in the gap may be increased. However this approach has been considered to have the undesirable consequence of reducing the effective optical path length by a proportion similar to the increase of the magnetic field [19]. Therefore the perception was that the same value of $B L_{\mathrm{opt}}$ product would result regardless of the size of the gap and the eventual rotation angle would be improved very little by altering the geometrical dimensions of the concentrator. As a result, the gap size has not in the past been an important design parameter [19]-[22] with its choice based on merely the consideration of fabrication convenience [19], [21].

In this paper, we examine the problem of a light beam's passage through a bulk optical glass. It is found that the optical path length, $L_{\mathrm{opt}}$, can be made to remain the same regardless of the thickness of the bulk glass, rather than a monotonic function of the glass thickness as previously believed [19]. This suggests that if the magnetic field is increased by means of a smaller gap size, the $B L_{\mathrm{opt}}$ product would be increased proportionally for the same electric current and this should lead to an improved sensitivity of the optical current sensor. To explore this new sensitivity enhancement technique, we develop a simple mathematical model for a systematic analysis of its possible benefits and limitations as well as its feasibility in practical devices. It is shown that even with practicalities, such as optical power attenuation, taken into account, the proposed small gap configuration could still lead to an appreciable 

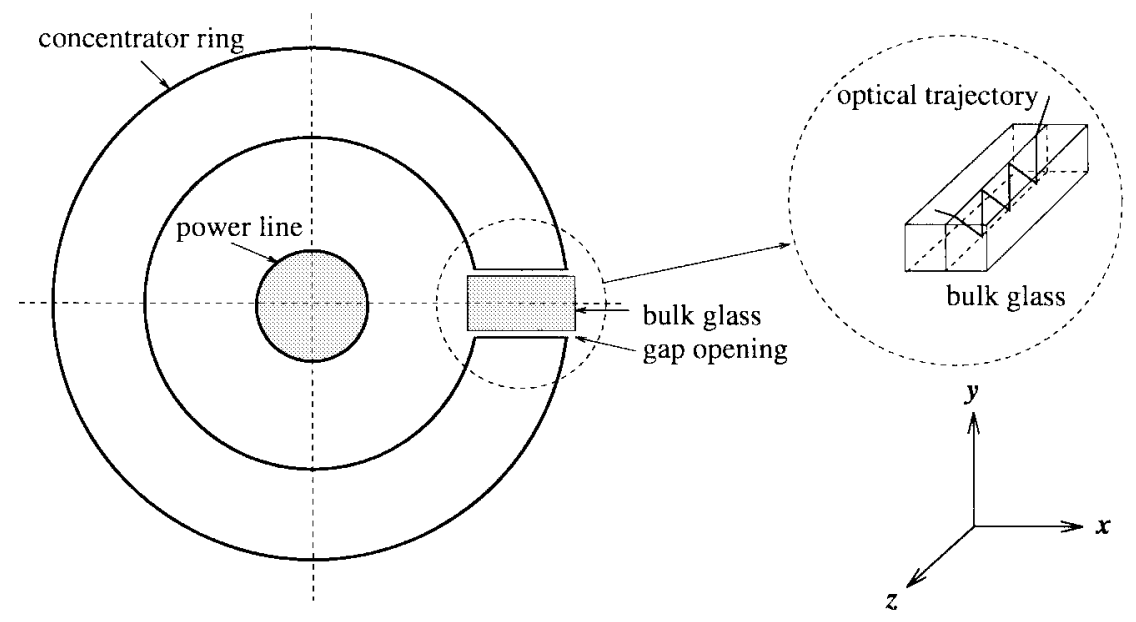

Fig. 1. Field concentrator arrangement for current measurement.

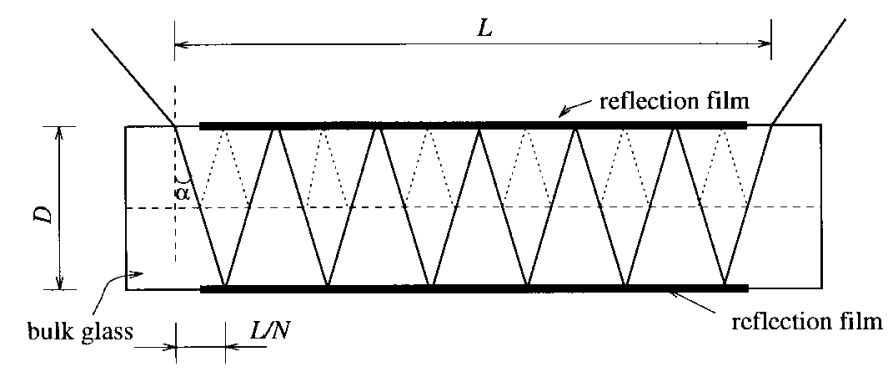

Fig. 2. Optical trajectory in a bulk glass of thickness $D$ (solid line) and its counterpart in a comparison glass of halved thickness (dashed line).

amount of enhancement in device sensitivity. A bulk-glass optical current sensor with a field concentrator is then designed and tested to confirm experimentally the proposed sensitivity improvement technique.

\section{Conservation of the Optical Path Length}

After an optical beam is fed into a bulk glass sensing element, it usually undergoes a multiple reflection trajectory as shown in Fig. 2. If the gap in the concentrator is reduced in size, the thickness of the bulk glass needs to be reduced as well so as to fit into the smaller gap. Suppose we consider the case where the glass thickness is halved. The corresponding change to the optical trajectory may be drawn as in Fig. 2 simply by folding the bottom half of the original trajectory upon the top half. By comparing the original and the new trajectories in Fig. 2, it is clear that their optical lengths are the same.

In general, any amount of reduction in the glass thickness leads to two changes of the optical trajectory, namely, an increase in the number of reflections, and a reduction in the elemental optical length which is the trajectory length between two adjacent reflections. Intuitively, the increase in the number of reflections may be expected to be inversely proportional to the elemental optical length, no matter how much the glass thickness is reduced. In other words, when these two changes are taken into account, their contributions to the total optical length should cancel each other. Here, we employ a simple derivation to verify the above suggestion.
Consider the case where the glass is immersed in a vertically directed magnetic field. Suppose we use $N$ to denote the total number of reflections. The vertical component of the elemental optical length is simply the glass thickness, $D$, which for a large $N$ relates approximately to the length of the bulk glass, $L$, by

$$
D=\frac{L / N}{\tan \alpha}
$$

where $\alpha$ is the incident angle of the optical beam in the glass (see Fig. 2). The total effective optical path length, $L_{\mathrm{opt}}$, is the component of the total optical trajectory length in the direction of the magnetic field, which in this case is the vertical direction. Thus

$$
L_{\mathrm{opt}}=N \times D=L / \tan \alpha .
$$

This suggests that although $L_{\mathrm{opt}}$ is not continuously invariant since $N$ is an integer, it is independent of the glass thickness. The main implication of (3) is that a reduction in the elemental optical length is fully compensated by the resultant increase in the number of reflections. Thus for a given glass and a given angle at which the optical beam is fed into the glass, the optical length is a constant. The above finding is of paramount importance for the design of Faraday optical current sensors with $B$-field concentrators. Because of the conservation of the optical length, any increase of the magnetic field by reducing the gap opening, or indeed any alteration of the geometrical dimensions of the concentrator, can benefit at no expense to the optical length. This should lead to a substantial improvement of device sensitivity of Faraday optical current sensors.

\section{Gap Size Dependence of the Magnetic Field}

The calculation of the magnetic field in the concentrator gap may be simplified by assuming that the magnetic flux in both the concentrator and its gap opening is confined to purely circular paths as in the case of a closed concentrator ring. This implies a negligible leakage flux and thus a one-dimensional equivalent magnetic circuit may be constructed along any concentric circle within the concentrator. In Fig. 3(a), a concentric circle of the average radius of the concentrator ring is drawn, 


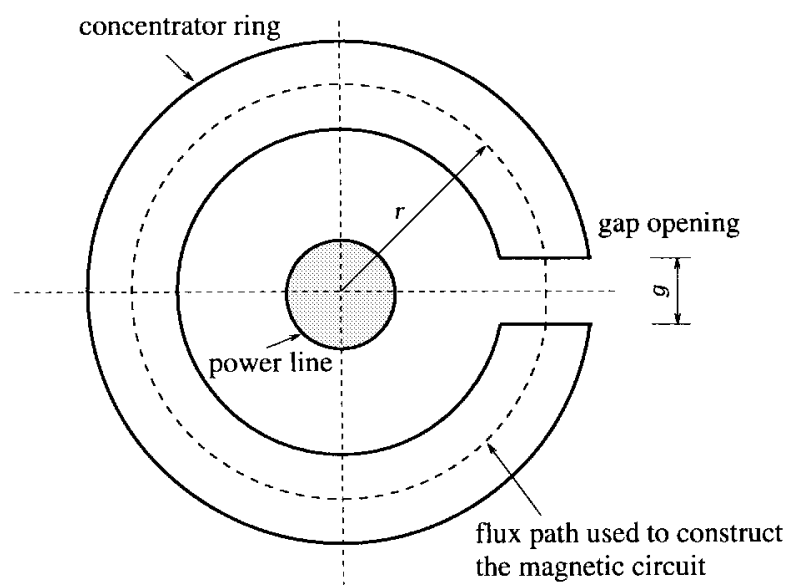

(a)
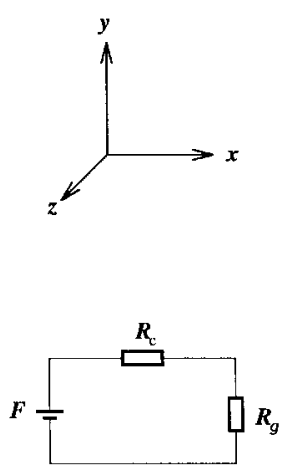

(b)

Fig. 3. Modeling the concentrator ring (a) with an equivalent magnetic circuit (b).

and its magnetic circuit is illustrated in Fig. 3(b). Here $F$ is the magnetomotive force (mmf) and $R_{c}, R_{g}$ are the reluctances of the ring and the gap, respectively, given by [25]

$$
\begin{aligned}
& R_{c}=\frac{2 \pi r-g}{\mu_{0} \mu_{r} A_{0}} \\
& R_{g}=\frac{g}{\mu_{0} A_{0}}
\end{aligned}
$$

with $A_{0}$ being the cross sectional area of the concentrator, $g$ being the size of the gap opening, and $\mu_{r}$ being the relative permeability of the ferromagnetic material of the concentrator.

Note that in a magnetic circuit, the circuit current corresponds to the magnetic flux, $\Phi$. Thus, from Fig. 3(b), the magnetic flux may be formulated as

$$
\Phi=\frac{F}{R_{c}+R_{g}}=\frac{\mu_{0} \mu_{r} A_{0} F}{2 \pi r+\left(\mu_{r}-1\right) g}
$$

where $F=N_{c} I_{c}$ with $N_{c}$ being number of turns around the concentrator ring and $I_{c}$ the current in the coil. Thus the magnetic field at the centre of the concentrator gap is given by

$$
B_{g} \equiv \frac{\Phi}{A_{0}}=\frac{\mu_{0} \mu_{r} F}{2 \pi r+\left(\mu_{r}-1\right) g} .
$$

For convenience of comparison, the gap magnetic field is normalized with respect to some reference gap size, $g_{0}$ so that the magnetic field may be rewritten in the following normalized form:

$$
\bar{B}_{g}=\frac{2 \pi r+\left(\mu_{r}-1\right) g_{0}}{2 \pi r+\left(\mu_{r}-1\right) g} .
$$

To illustrate its dependence on the gap size, the magnetic field at the gap centre is estimated for a practical OCS [19], [21] for which the following parameters are used:

$$
r=28 \mathrm{~mm}, \quad g_{0}=10 \mathrm{~mm}, \quad \text { and } \mu_{r}=1000 .
$$

Equation (7) is used to calculate the magnetic field generated, and then compared to that from a dedicated 2-D finiteelement electromagnetic code, Opera-2D [26], to validate the applicability of the one-dimensional circuit model. The comparison presented in Fig. 4 shows that an excellent agreement is observed between the equivalent circuit model and the

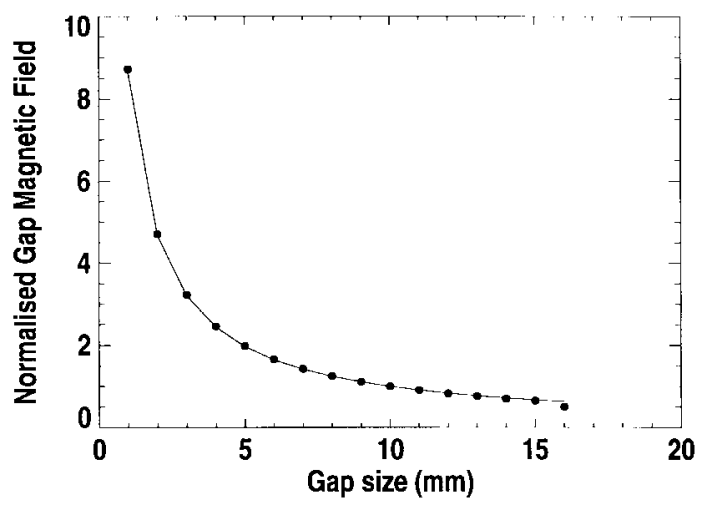

Fig. 4. Normalized magnetic field calculated with the magnetic circuit model (solid line) and the computer package (dots).

computer code for all gap sizes but $g=16 \mathrm{~mm}$ which appears to be too large to support the assumption of a negligible leakage flux. Therefore for gap sizes below $16 \mathrm{~mm}$ the gap size dependence of the magnetic field is the simple reciprocal function in (7). Fig. 4 also suggests that a reduction of the gap size from 10 to $5 \mathrm{~mm}$ would lead to the magnetic field being doubled. This may be translated into a two-fold increase of the eventual Faraday rotation angle and hence approximately the same amount of enhancement in the OCS's sensitivity, clearly a significant improvement of device performance.

\section{EFFECTS OF OPTICAL POWER LOSSES}

As established above, the optical path length is independent of the glass thickness. One implication is that the internal power loss of the optical beam within the bulk glass, due to the infrared absorption of the glass material for instance, should also be invariant. However there will be optical power losses due to the reflection of the optical beam on the interfaces between the bulk glass and its reflection films. Possible causes of this "external" power loss may include the surface roughness of the reflection film and an inadequate film thickness for total reflection. An optical beam passing through a thin glass has more reflections than that through a thick glass and thus it may experience more power losses in the former case compared with the latter. As a result, the increased $B L_{\mathrm{opt}}$ product with 
a smaller concentrator gap may not be fully beneficial. The effect of the external power loss therefore needs be assessed.

Suppose after a single reflection of an optical beam, its power retains a fraction of $\eta$ of that before reflection $(\eta \leq 1)$. Then, if we assume that at the reference glass thickness $D_{0}$ the total reflection number is $N_{0}$, the transmission coefficient of the optical beam through the bulk glass is

$$
\mathcal{T}_{0}=\eta^{N_{0}} .
$$

Similarly if there are $N$ reflections for a glass thickness $D$, the transmission coefficient becomes

$$
\mathcal{T}=\eta^{N}=\left(\eta^{N_{0}}\right)^{N / N_{0}}=\mathcal{T}_{0}^{N / N_{0}} .
$$

After the polarization state of an optical beam is Faraday rotated, the light intensity measured with an analyzer at $45^{\circ}$ with respect to the polarizer is reduced to [19]

$$
I_{f}=\frac{1}{2} I_{0}(1-\sin 2 \theta)
$$

where $I_{0}, I_{f}$ are the light intensity measured after the optical beam's passage through the polarizer and the analyzer, respectively, and $\theta$ is the Faraday rotation angle. The above equation does not take into account the optical power losses both within the bulk glass material and at the reflection films. When these effects are considered, (10) becomes

$$
I_{f}=\frac{1}{2} \mathcal{T} \mathcal{T}_{i}(1-\sin 2 \theta) I_{0}
$$

where $\mathcal{T}_{i}$ is the power transmission coefficient due to the internal optical losses. The relative light intensity change

$$
\Delta \mathcal{P}_{R} \equiv \frac{I_{f}}{I_{0}}-0.5=\frac{1}{2} \mathcal{T} \mathcal{T}_{i} \sin 2 \theta
$$

is used as a measure of the Faraday rotation angle around the device's usual operating point of $I_{f} / I_{0}=0.5$. Since device sensitivity may be defined as the ratio of the light intensity change to the current change in the power line, $\Delta \mathcal{P}_{R}$ is also a measure of device sensitivity. Therefore to compare device sensitivity for the two cases of interest, one may measure the improvement of the relative light intensity change by using the sensitivity ratio defined as

$$
\mathcal{R}_{s} \equiv \frac{\Delta \mathcal{P}_{R}}{\Delta \mathcal{P}_{R 0}}=\mathcal{T}_{0}^{N / N_{0}-1} \frac{\sin 2 \theta}{\sin 2 \theta_{0}}
$$

where the contribution of the internal power loss is eliminated as it is a constant for both cases.

To illustrate the performance of the reduced gap technique, (13) is applied to the case where the Faraday rotation angle is small such that $\sin 2 \theta \approx 2 \theta$. With (1), (2), (3) and (7), this approximation reduces (13) to

$$
\mathcal{R}_{s}=\left[\frac{2 \pi r+\left(\mu_{r}-1\right) D_{0}}{2 \pi r+\left(\mu_{r}-1\right) D}\right] \mathcal{T}_{0}^{D_{0} / D-1}
$$

where $D_{0}=g_{0}$ and $D=g$ has been used. Once again, the parameters used to produce Fig. 4 are used to calculate the

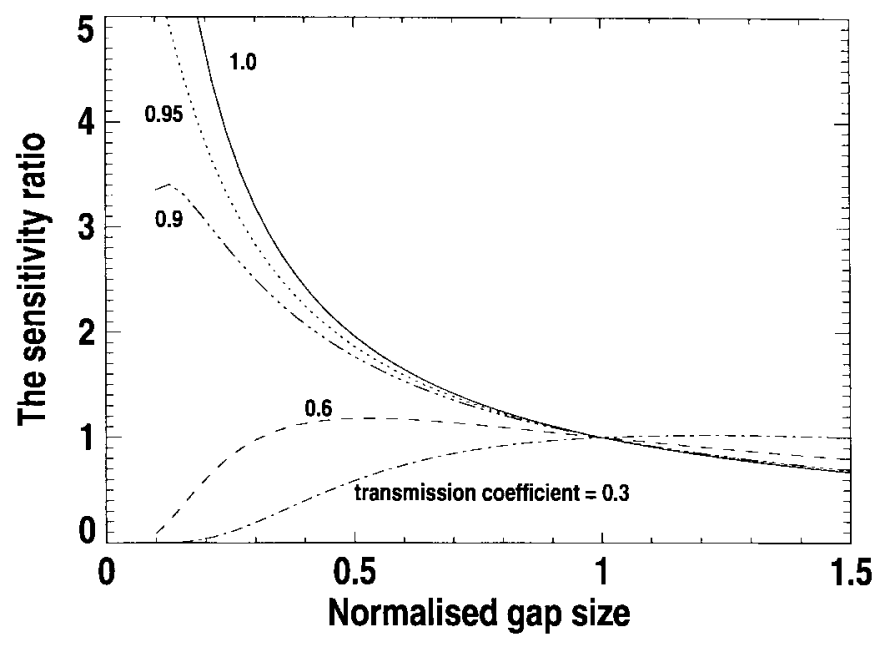

Fig. 5. The sensitivity ratio as a function of gap size normalized to a reference gap size $g_{0}$ at various transmission coefficients.

sensitivity ratio as a function of the gap size and the results are plotted in Fig. 5.

Fig. 5 suggests that for a reflection film coating that allows an external optical transmission of less than $60 \%$, there is little merit in reducing the gap size. In fact, it may be slightly advantageous to choose a large gap opening for cases where the external optical transmission coefficient becomes less than $30 \%$. This is because for these cases the external power loss is the predominate factor and it is necessary to ensure this is reduced by all means including an appropriate increase in glass thickness. On the other hand, once a good quality coating of the reflection films is achieved, the benefit of reducing the gap size overcomes the contribution of the external power loss. Fig. 5 shows that for an optical power transmission above $95 \%$ the device sensitivity is improved by nearly $90 \%$ when the gap size is reduced from $g_{0}$ to $0.5 g_{0}$. It is worth mentioning that with the bulk glass coated with a dielectric film it is possible to achieve a total reflection of light beam at the critical incident angle [22], [29] and therefore a perfect optical power transmission is feasible in principle. On the other hand, it is also possible for conducting coatings, such as metal films, to achieve a power transmission coefficient as high as 95\% [19], [22]. Therefore the high power transmission coefficient and hence the large sensitivity enhancement projected in Fig. 5 are realistic possibilities

It should be emphasized that although the $95 \%$ optical power transmission may appear to be rather demanding as far as the device fabrication is concerned, this figure refers to the contribution of the reflection loss of the optical power only. Therefore even if the power absorption of the glass material is high at some operational wavelengths, the aforementioned 90\% improvement of device sensitivity is still a valid deduction. In other words, it is concerned with the quality of the reflection films rather than the absorption characteristics of the glass material itself. Any reduction in the internal loss of the optical power will improve the absolute value of intensity measured after the $45^{\circ}$ analyzing polarizer and hence reduce the minimum measurable current of the device. However it does not improve the enhancement of device sensitivity. 


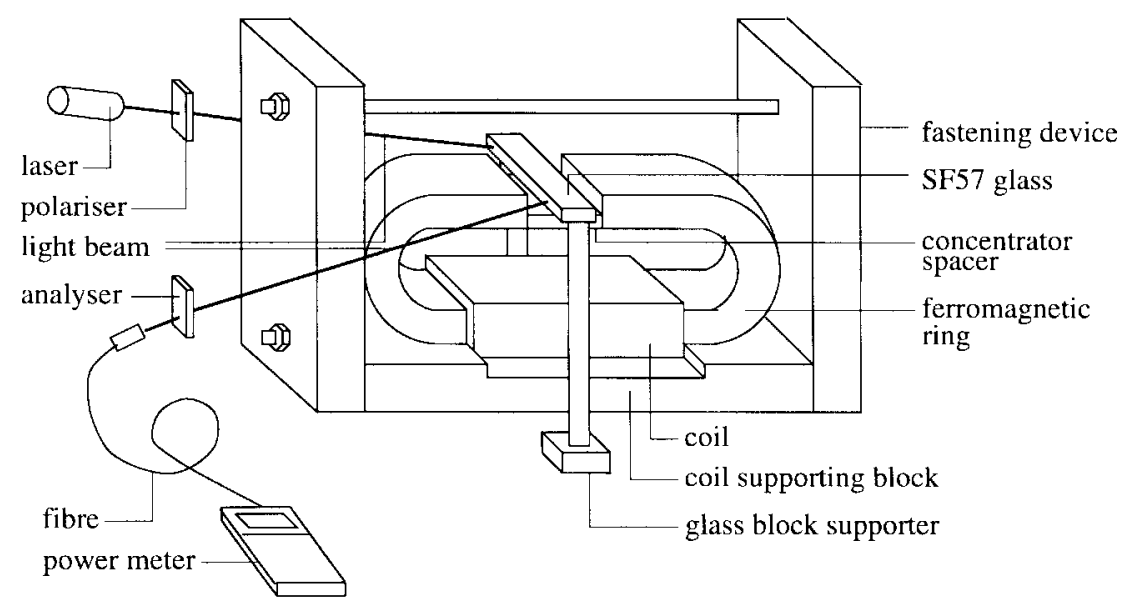

Fig. 6. Schematic illustration of the experimental system.

Apart from various sources of power losses in the bulk glass, an optical beam is also subject to a possible phase difference between its two orthogonal electric field components when it is reflected at reflection coatings [22], [29]. This often leads to an additional rotation of its polarization state, making it difficult to determine Faraday rotation accurately in experiments [22], [29]. It is of interest to note that at zero Faraday rotation a linearly polarized optical beam has only one electric field component (parallel to the reflection film) and as such its polarization state should remain unaffected even though a phase shift may be induced after light reflection [22], [29]. As the Faraday rotation is raised gradually from zero however, the initially parallel (to the reflection film plane) field component starts to rotate as well, and this causes the light beam to acquire an additional electric field component that is perpendicular to the reflection coating plane. As a result, the polarization state of the light beam is distorted after it undergoes the next reflection from the reflection film. The amount of this reflection-induced rotation of polarization state depends on the actual incident angle of the light beam and the ratio of its two electric field components, the latter of which in turn depends on Faraday rotation executed between each of its two adjacent reflections. Therefore it is difficult to generalize such an effect for incorporation into our analytical treatment of light propagation in bulk glass [see (11)]. In qualitative terms however, it is conceivable that a large Faraday rotation is likely to induce a sizeable polarization distortion. For practical optical current sensors, their Faraday rotation angle is usually limited to $\pm 2^{\circ}$ to satisfy the high sensitivity requirements of the power industry [19] and as a result they should have a reasonably small reflection-induced polarization distortion. Note also that such a distortion is generally very small for well designed optical current sensors where precautions are often taken for its reduction or even elimination [30]. Consequently, the analytical formulation of device sensitivity from (12) to (14) should be applicable approximately to practical devices.

The analysis based on Fig. 5 applies to cases where the Faraday rotation angle is small. This may correspond to relatively low current measurements. If the absolute rotation angle is adequately measurable, the enhancement of the $B L_{\mathrm{opt}}$ product may be used to compensate for a smaller Verdet constant so that alternative glass materials can be considered to improve other aspects of device performance. For cases where the absolute rotation angle is already appreciable $\left(\theta \geq 10^{\circ}\right)$, the sensitivity improvement may be not as good as predicted in Fig. 5 since the assumption $\sin 2 \theta \approx 2 \theta$ overestimates $\sin 2 \theta$ for large $\theta$.

\section{EXPERIMENTAL ARRANGEMENT}

To examine the feasibility of the proposed sensitivity enhancement scheme, a bulk-glass optical current sensor with a field concentrator was studied using the experimental arrangement shown in Fig. 6. The concentrator was constructed from two separated C-shaped ferromagnetic rings, and as such it has two gap openings with the first for insertion of the bulk optical glass and the second located in the middle of the coil winding (see Fig. 6). As a result, this field concentrator is likely to have more magnetic flux leakage at a given coil current than the field concentrator used in commercial devices as the latter is essentially a single-piece C-shaped ferromagnetic ring [19]-[22]. On the other hand, its construction using two separated ferromagnetic rings implies that the magnetic attraction force induced between the two rings at finite coil current would cause them to move toward each other, leading to a significant change of the gap field. To minimize such a movement, the two rings were separated by plastic spacers at both concentrator gaps and a fasten device was also introduced to hold the two rings tightly together (see Fig. 6). Such a concentrator arrangement may appear to be complicated compared to the usual single ferromagnetic ring arrangement [19]-[22]. However, it has the advantage of being able to change easily the gap size by using differently sized plastic spacers, and this allows experiments for different gap sizes to be performed with essentially the same field concentrator.

In the experimental arrangement in Fig. 6, a $\mathrm{He}-\mathrm{Ne}$ laser was used to provide a light beam at $632.8 \mathrm{~nm}$, and a polarizer was placed after the $\mathrm{He}-\mathrm{Ne}$ laser to convert the light beam into a linearly polarized one. This linearly polarized light beam was then launched into the sensing element (a glass block) to undergo a multiple reflection trajectory (see Fig. 2) with an 
incident angle within the glass adjusted at $\alpha=33.0^{\circ}$. The light beam leaving the glass block passed through an analyzer at an angle of $45^{\circ}$ with respect to the polarizer before it was measured by a power meter. A SF57 glass block was used as the sensing element, because of its high Verdet constant [27], [28] and its negligibly small temperature dependence [28]. At the wavelength of the light beam $(632.8 \mathrm{~nm})$, its Verdet constant is $21.8 \mathrm{rad} / \mathrm{T} \cdot \mathrm{m}$ [27]. The central section of the glass block was coated with a thin aluminum layer as the reflection film, and with $\alpha=33.0^{\circ}$ the optical path length was found to be $L_{\mathrm{opt}}=10.25 \mathrm{~cm}$. To ensure that it is held properly in position at the gap centre free from being affected by possible concentrator movement, the glass block was not supported by the plastic spacer at the gap but by a pair of plastic supporting blocks outside the concentrator (see Fig. 6).

\section{EXPERIMENTAL RESULTS}

The magnetic field between the two $\mathrm{C}$-shaped ferromagnetic rings was measured using a Hall probe with the glass block removed from the concentrator, and its dependence on coil current is shown in Fig. 7 for two gap sizes, 10 and 20 $\mathrm{mm}$. It is of interest to note that with the coil current below 3 A the gap magnetic field has an approximately linear dependence on coil current. As the coil current increases, the field-current relationship starts to deviate from the linear dependence, indicating possible onset of flux saturation in the field concentrator. Within the limit of the linear dependence, the gap magnetic field was found to be 0.066 and $0.102 \mathrm{~T}$ at a coil current of 2 A for $g=20 \mathrm{~mm}$ and $g=10$ $\mathrm{mm}$, respectively, corresponding to a nominal Faraday rotation angle of 8 and $13^{\circ}$ calculated from (1) with $V=21.8 \mathrm{rad} / \mathrm{T} \cdot \mathrm{m}$ and $L_{\text {opt }}=10.25 \mathrm{~cm}$. The increment in gap magnetic field by halving the gap size is found to be about $53.5 \%$ at this coil current $(2 \mathrm{~A})$. However, according to the theoretical model developed for a single-ring concentrator in Section III, the magnetic field should be increased by $100 \%$ when the gap size is halved. This reduction in experimental field increment from its theoretical value is caused by the additional magnetic flux leakage at the second gap of the two-ring concentrator. Although a reformulation of the theoretical model for the two ring concentrator is feasible, it is only of secondary importance for this study since the main interest here is to examine the dependence of measured Faraday rotation upon measured magnetic field.

With the dependence of the gap field on the coil current measured, the glass block was placed at the gap centre. The output light intensity $I_{f}$ was first measured at zero coil current and compared with the incident light intensity $I_{0}$ for two gap opening cases $(10 \mathrm{~mm}$ and $20 \mathrm{~mm})$. It was found that $I_{f} / I_{0}$ was about 0.5 with a relative error around 0.2 and $0.5 \%$ for a gap size of 20 and $10 \mathrm{~mm}$, respectively. This light intensity measurement was repeated for various coil current values up to $15 \mathrm{~A}$ and then used to derive the Faraday rotation angle from (10). In Fig. 8, a relative light intensity change, $I_{f} / I_{0}-0.5$, is plotted as a function of coil current. Since the device sensitivity is proportional to the relative light intensity change (12), it is apparent from Fig. 8 that the

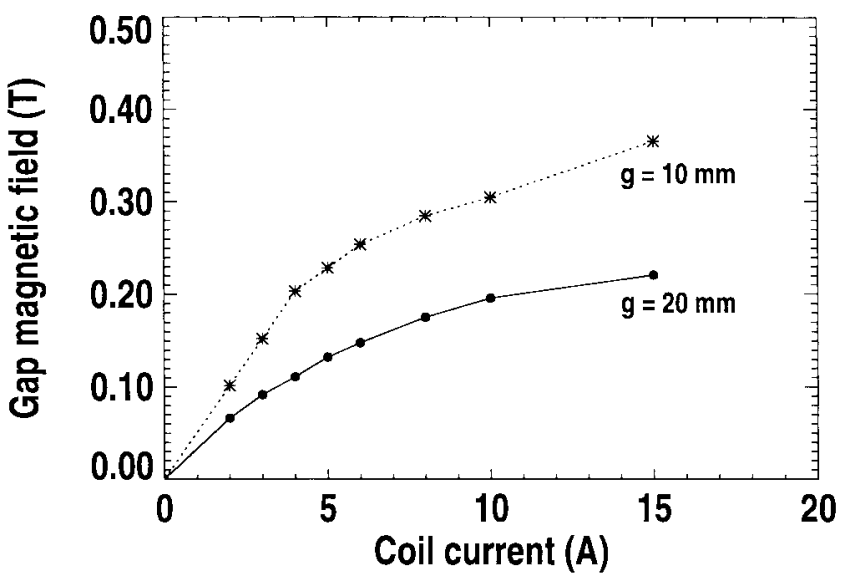

Fig. 7. Magnetic field measured at the gap centre as a function of coil current.

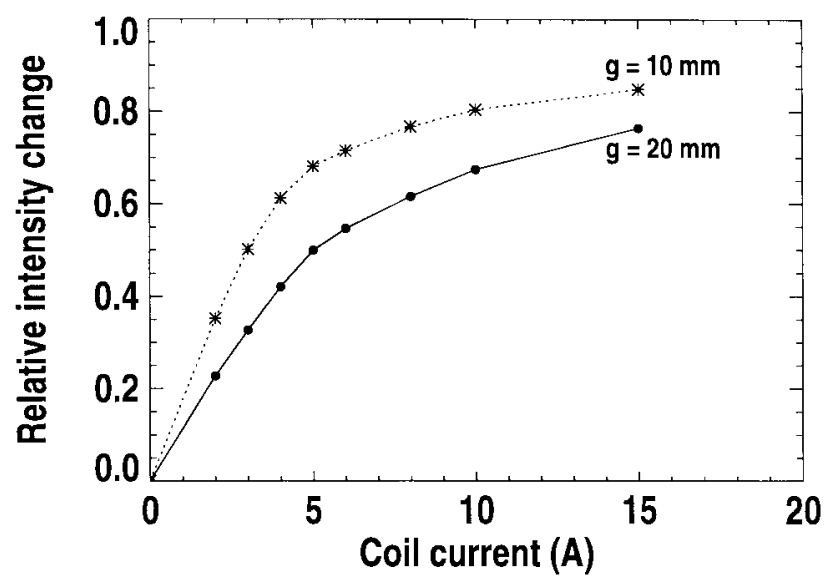

Fig. 8. Relative output optical intensity $\left(I_{f} / I_{0}-0.5\right)$ as a function of coil current at two gap opening sizes.

small gap configuration always has a greater device sensitivity than the large gap configuration. The coil current dependence of the relative light intensity change resembles qualitatively that of the gap magnetic field in Fig. 7, suggesting a close correlation between the device sensitivity enhancement and the gap field increment. Therefore a large gap magnetic field does lead to an improvement of device sensitivity. Around the operation point of $I_{f} / I_{0}=0.5$, the device sensitivity improvement may be assessed by estimating the increment in the slopes of the two curves at zero coil current. Calculation shows that the slope increment (and thus the device sensitivity increment) is about $54.9 \%$. This is in excellent agreement with the measured magnetic field increment of 53.5\% around zero coil current. An interesting deduction from this close agreement is that the reflection-induced polarization distortion discussed in the preceding section appears to be negligibly small for coil currents below $3 \mathrm{~A}$, and this confirms our earlier suggestion that the polarization distortion is small within the usual operation range of $\pm 2^{\circ}$ Faraday rotation. However, at larger coil current, the device sensitivity improvement becomes less correlated to the magnetic field increment, most likely due to the nonlinearity of the $\cos ^{2} \theta$ dependence of $I_{f} / I_{0}$. It may also be partly due to the reflection induced polarization distortion discussed above. 


\section{CONCLUSION}

In this contribution, we have proposed a technique to enhance the Faraday rotation angle for one class of bulk glass optical current sensors at a given electric current by reducing the gap opening of the field concentrator. A simple analytical model has been suggested, from which device sensitivity was formulated and assessed in terms of its dependence upon the optical path length, the gap magnetic field, and various optical power losses in the sensing optical glass. In addition, effects of possible reflection-induced polarization distortion have been discussed and assessed. With a systematic consideration of the above factors, it has been established theoretically that, because of the conservation of the optical path length, advantage can be taken of the increased magnetic field to improve device sensitivity significantly. These suggestions have been confirmed with a detailed experimental study. The basic principle of the proposed technique may be used to improve the device sensitivity substantially or alternatively to compensate a smaller Verdet constant so that a wider range of glass materials may be utilized for exploring improvements with respect to other aspects of device performance.

\section{REFERENCES}

[1] R. E. Hebner, R. A. Malewski, and E. C. Cassidy, "Optical methods of electrical measurement at high voltage," in Proc. IEEE, 1977, vol. 65, pp. $1524-1548$.

[2] A. M. Smith, "Polarization and magnetooptic properties of single-mode optical fiber," Applied Optics, vol. 17, pp. 52-56, 1978.

[3] A. J. Rogers, "Optical methods for measurement of voltage and current on power systems," IEE J. Electron. Power Appl., vol. 2, pp. 120-124, 1979.

[4] A. Papp and H. Harms, "Magnetooptical current transformer 1: Principles," Appl. Opt., vol. 19, pp. 3729-3734, 1980.

[5] G. W. Day and A. H. Rose, "Faraday effect sensors: The state of art," in Proc. SPIE, 1988, vol. 985, pp. 138-150.

[6] G. W. Day, M. N. Deeter, and A. H. Rose, "Faraday effect sensors: A review of recent progress," in Advances in Optical Fiber Sensors, B. Culshaw, E. L. Moore, and Z. Zhipeng, Eds. SPIE Press, 1992.

[7] IEEE Optical Sensor Review Committee, "Optical current transducers for power-systems-A review," IEEE Trans. Power Delivery, vol. 9, pp. 1778-1788, 1994.

[8] Y. N. Ning, Z. P. Wang, A. W. Palmer, K. T. V. Grattan, and D. A. Jackson, "Recent progress in optical current sensing techniques," Rev. Sci. Instrum., vol. 66, pp. 3097-3111, 1995.

[9] S. C. Rashleigh, "Magnetic field sensing with a single-mode fiber," Opt. Lett., vol. 6, pp. 19-21, 1981.

[10] R. I. Laming and D. N. Payne, "Electric-current sensors employing spun highly birefringent optical fibers," J. Lightwave Technol., vol. 7, pp. 2084-2094, 1989.

[11] D. D. Tang, A. H. Rose, G. W. Day, and S. M. Etzel, "Annealing of linear birefringence in single-mode fiber coils-Application to optical fiber current sensors," J. Lightwave Technol., vol. 9, pp. 1031-1037, 1991.

[12] I. G. Clarke, "Temperature-stable spun elliptic-core optical-fiber current transducer," Opt. Lett., vol. 18, pp. 158-160, 1993.

[13] N. C. Pistoni and M. Martinelli, "Vibration-insensitive fiber-optic current sensor," Opt. Lett., vol. 18, pp. 314-316, 1993.

[14] A. J. Rogers, J. C. Xu, and J. L. Yao, "Vibration immunity for optical-fiber current measurement," J. Lightwave Technol., vol. 13, pp. 1371-1377, 1995.

[15] X. Fang and R. O. Claus, "Optimal design of IRIS-based polarimetric intrinsic fiber optic current sensors," J. Lightwave Technol., vol. 9 pp. 1664-1673, 1996.

[16] E. A. Ulmer Jr., "A high-accuracy optical current transducer for electric power systems," IEEE Trans. Power Delivery, vol. 5, pp. 892-898, 1990.

[17] Y. N. Ying, B. C. B. Chu, and D. A. Jackson, Opt. Lett., vol. 16, pp. 1996-1998, 1991

[18] B. C. B. Chu, Y. N. Ning, and D. A. Jackson, Opt. Lett., vol. 17, pp. $1167-1169,1992$
[19] N. A. Pilling, "Optical fiber measurement in power systems," Ph.D. dissertation, Univ. of Liverpool, England, ch. 4, 1992.

[20] Y. Yamagata, T. Oshi, H. Katsukawa, S. Kato, and Y. Sakurai, "Development of optical current transformers and application to fault location systems for substations," IEEE Trans. Power Delivery, vol. 8, pp. 866-873, 1993

[21] K. G. Lewis, R. E. Jones, and G. R. Jones, in Proc. 2nd Int. Conf Reliability of Transmission and Distrib. Equipments, Warwick, UK, Mar. 1995.

[22] J. Song, P. G. McLaren, D. J. Thomson, and R. L. Middleton, "A prototype clamp-on magneto-optical current transducer for power system metering and relaying," IEEE Trans. Power Delivery, vol. 10, pp. 1764-1770, 1995.

[23] P. R. Former and F. C. Johoda, "Linear birefringence effects on fiberoptic current sensors," Appl. Opt., vol. 27, pp. 3088-3096, 1988.

[24] E. Hecht, Optics, 2nd ed. Reading, MA: Addison-Wesley, 1987, ch. 8

[25] R. L. Boylestad, Introductory Circuit Theory, 5th ed. Columbus, $\mathrm{OH}$ Merrill, 1987, pp. 313-315.

[26] Vector Field Ltd., 24 Bankside, Kidlington, Oxford OX5 1JE, England.

[27] G. Westenberger, H. J. Hoffmann, W. W. Jochs, and G. Przybilla, "The Verdet constant and its dispersion in optical glasses," in Proc. SPIE, 1991, vol. 1535 , pp. $113-120$.

[28] P. A. Williams, A. H. Rose, G. W. Day, T. E. Miller, and M. N. Deeter, "Temperature dependence of the Verdet constant in several diamagnetic glasses," Appl. Opt., vol. 30, pp. 1176-1178, 1991.

[29] J. M. Bennett, "Polarization," in Handbooks of Optics, 2nd ed., M. Bass, E. W. van Stryland, D. R. Williams, and W. L. Wolfe, vol. 1, pp. 5.1-5.20, 1995 .

[30] Y. Yoshino, Y. Takahashi, M. Gojyuki, and T. Shimoyama, "Polygonal Faraday effect current sensor with polarization-preserving dielectric mirrors," in Proc. SPIE, 1994, vol. 2292, pp. 34-41.

Gongde Li (S'95) received the B.Sc. and M.Sc. degrees in photo-electronic engineering from Changchun Institute of Optics and Fine Mechanics, People's Republic of China, in 1982 and 1985, respectively. Since 1993, he has been working towards the Ph.D. degree at the University of Liverpool, UK, and his dissertation work is concerned with novel optical fiber sensing techniques for power system applications.

From 1985 to 1992, he was employed at the Changchun Institute of Optics and Fine Mechanics, where he worked in the field of optical design for laser scanning instrumentation systems and optoelectronic measurement systems.

Michael G. Kong (M'94) received the B.Sc. and M.Sc. degrees in physical electronics from Zhejiang University, People's Republic of China, in 1984 and 1987, respectively. He received the Ph.D. degree in electrical engineering from Liverpool University, UK, in 1992.

From 1992 to 1994 , he worked as a postdoctoral research fellow on a compact free-electron laser project and a computational plasma project at the University of Liverpool and the University of Nottingham, UK, respectively. In 1995, he returned to the University of Liverpool as a Lecturer. His present research interests include optical sensors, free-electron lasers, optical diagnostics techniques for arc plasma and material processing plasma.

Gordon R. Jones has conducted research in optical monitoring techniques for many decades at the University of Liverpool, UK, where he is a Professor of Electrical Engineering. He is a member of an international study group on switch gear arcs and chairs their specialist group on arc measurement, diagnostics, and monitoring. He is the Director of the Centre for Intelligent Monitoring Systems at Liverpool funded by the European Union.

Dr. Jones is a Fellow of the Institution of Electrical Engineers (IEE).

Joe W. Spencer is actively involved with one of the IEE professional groups and with a number of international committees He is also actively involved in research specializing in the application of novel optical fiber-based sensors and instrumentation. In particular, his research is directed towards the monitoring of industrial plant, where the environmental conditions are severe (e.g., electricity generation and distribution plant, nuclear plant, and gas turbines used for electric power generation and for aircraft propulsion.) He has been an Invited Lecturer in both China and Japan.

Dr. Spencer is a member of the Institution of Electrical Engineers (IEE) and a Chartered Engineer (C.Eng.) 